\title{
ANOVA-SRC-BPSO: a hybrid filter and swarm optimization-based method for gene selection and cancer classification using gene expression profiles
}

\author{
Salim Sazzed \\ Old Dominion University, Norfolk, VA, USA
}

\begin{abstract}
Gene expression profiling reveals the activity of thousands of genes that can help to identify cancer biomarkers. However, the presence of such a large number of genes in the profiles inflicts a high computational burden on classifiers. To deal with the high-dimensional feature space, in this paper, we introduce a 3-phase feature selection framework, ANOVA-SRC-BPSO. ANOVA-SRC-BPSO first distinguishes the highly class-correlated genes utilizing the analysis of variance (ANOVA) and F-test. In the second phase, we employ Spearman rank-order correlation (SRC) to eliminate redundant genes. Finally, we leverage the binary particle swarm optimization (BPSO) with the support vector machine (SVM) classifier to select an optimized feature subset. We report the accuracy of ANOVA-SRC-BPSO utilizing the SVM classifier in seven gene expression datasets. The comparisons with fourteen state-of-the-art methods show that ANOVA-SRC-BPSO yields the highest accuracy in five datasets. Moreover, we disclose that the performances of various feature selection approaches are inconsistent across gene expression datasets.
\end{abstract}

Keywords: cancer classification, gene selection, PSO, microarray cancer, evolutionary algorithm, metaheuristic optimization, binary particle swarm optimization

\section{Introduction}

Cancer classification utilizing gene expression profiles reveals the underlying causes relating to cancer and helps early diagnosis of cancer and drug discovery. The microarray gene expression profiles contain a high number of genes with a small sample size, which makes cancer classification very challenging (feature selection is an NP-hard problem [1]). Besides, among the thousands of genes, only a few of them are useful for classification. Identifying informative genes is a crucial step for cancer classification, as it allows researchers to identify the most substantial genes that provide insight into the mechanisms responsible for cancer. Furthermore, gene selection decreases the computational costs associated with the classification by excluding non-informative and redundant genes that increase the search space without adding any benefits. Besides, it improves the classification performance as shown by [2]. Furthermore, gene selection assists in introducing a simpler classification model, thus make the results more interpretable.

Researchers mainly utilized three approaches for gene selection, filter, wrapper, and hybrid approaches [2]. In the filter approach, the feature selection step is independent of the classification algorithm. A filter method estimates the relevance of features by computing statistical measures. Usually, a relevance score is calculated for every feature, and then low-ranked features are discarded; the remaining features are used for classification. The wrapper methods examine various combinations of gene subsets and determine the best subset by integrating them into a classifier. As they consider interactions among features during the best subset search, they are usually more accurate than the filter-based methods. However, due to the large search space, the wrapper methods are much slower than

*ssazz001@odu.edu

This article is (C) 2021 by author(s) as listed above. The article is licensed under a Creative Commons Attribution (CC BY 4.0) International license (https://creativecommons.org/licenses/by/4.0/legalcode), except where otherwise indicated with respect to particular material included in the article. The article should be attributed to the author(s) identified above. 
the filter-based methods. As the number of the genes increases, their computation time can grow exponentially. When multiple classifiers are employed for feature selection, the wrapper methods become even more computationally expensive. Furthermore, they are more prone to over-fitting [3].

The hybrid approaches combine the filter and wrapper-based methods and complement the benefits of both [4]. The hybrid methodologies enjoy the computational efficiency of the filter-based methods along with the high performance of wrapper-based approaches. The hybrid methods usually function in two steps; the first step reduces the feature space dimension using one or multiple filter-based methods. Afterward, a wrapper-based method is applied to select the optimal feature subset. Bio-inspired evolutionary algorithms have been widely used as wrapper methods for the hybrid approaches since they can obtain a near-optimal or sub-optimal solution exploring a reduced search space.

Here, we introduce a new hybrid gene selection approach that utilizes correlation measures and bio-inspired binary particle swarm optimization (BPSO) algorithm for cancer classification. The proposed methodology, ANOVA-SRC-BPSO, starts with identifying the top class discriminative genes using the filter-based ANOVA method. We utilize the Spearman rank-order correlation (SRC) coefficient to find and eliminate some class-correlated but redundant genes. Then, we apply the binary particle swarm optimization (BPSO) algorithm with the SVM classifier to identify an optimized feature subset. Finally, we employ the SVM classifier with the selected feature subset to classify cancer types. The comparisons with fourteen state-of-the-art methods on seven gene expression datasets show that ANOVA-SRC-BPSO yields the highest accuracy in five datasets.

\subsection{Motivation and Contributions}

The main objective of this paper is to demonstrate the effectiveness of the combination of ANOVA-SRC-BPSO and SVM for gene selection and cancer classification. Furthermore, we aim to show that although various methods exist for feature selection and cancer classification, none of them always yields better performance than others. The contributions of this work can be summarized as follows-

- We show that by employing ANOVA with F-test, the genes having high class correlation can be identified.

- We manifest that utilizing the Spearman rank-order correlation coefficient, it is possible to remove redundant genes.

- We attest that BPSO with the reduced feature set and SVM classifier can provide an optimized feature set that assists in achieving high accuracy.

\section{Background}

\subsection{Problem Statement}

The cancer classification problem can be formally described as follows:

Given a training set Train $=\left(s_{1}, c_{1}\right),\left(s_{2}, c_{2}\right), \ldots,\left(s_{\mathrm{n}}, c_{\mathrm{n}}\right)$, where each instance $s_{\mathrm{i}}$ represents a $d$-dimensional gene expression profile. When $d$ is the number of genes present in the dataset. $s_{\mathrm{i}}$ can be written as $=\left(g_{1}, g_{2}, \ldots, g_{\mathrm{d}}\right)$, where $g_{\mathrm{j}}$ represents gene expression value of a gene $j . \quad c_{i}$ refers the class label of the $i$ 'th instance and $c_{i} \in C$, where $C$ represents all classes.

Given a test set of size $m$, Test $=\left(s_{1}, s_{2}, \ldots, s_{m}\right)$ where each instance $s_{i}$ contains $d$ number of genes (same as the training set), the objective is to determine the class $c_{i}$ of the instance $s_{i}$. 


\subsection{Related Work}

Researchers employed univariate feature selection methods such as Chi-square [5], information gain (IG) [6, 7], Laplacian score (L-score) [8] and Fisher score (F-score) [9] for gene selection. Multivariate methods such as Minimal Redundancy Maximal Relevance (mRMR) [10], Random Subspace Method (RSM) [11] and Relevance Redundancy Feature Selection (RRFS) [12], which examine the dependencies among features have also been studied in literature.

The hybrid methodology initially employs a filter-based method to exclude non-informative genes to reduce search space. The RM-GA approach that combines ReliefF, mRMR, and genetic algorithm (GA) was proposed in [13]. In [14], the authors introduced a hybrid method named CFS-TGA, which utilized correlation-based feature selection (CFS), Taguchi-Genetic Algorithm (TGA), and K-NN classifier. In [15], the authors proposed a feature selection algorithm, mRMR, and combined it with the Artificial Bee Colony (ABC) algorithm to select informative genes. The performance of the mRMR-ABC algorithm was evaluated on six binary and multiclass gene expression microarray datasets employing the SVM classifier.

MIMAGA-Selection [16] is a hybrid feature selection algorithm that combines Mutual Information Maximization (MIM) and Adaptive Genetic Algorithm (AGA). CLA-ACO [17] is a hybrid approach that employs Cellular Learning Automata (CLA) and Ant Colony Optimization (ACO). CLA-ACO consists of three phases: filter-based Fisher criterion method, cellular learning automata, and ant colony optimization. In [18], the authors proposed IG/SGA, a feature selection method based on Information Gain (IG) and Standard Genetic Algorithm (SGA). Their method was evaluated on seven cancer microarray datasets and achieved 100\% accuracy in two datasets. A GA and Intelligent Dynamic Genetic Algorithm (IDGA) based method for gene selection was proposed in [19]. The proposed algorithm use Laplacian and Fisher scores independently in the first phase to select the top 500 genes. In the second phase, the reinforcement learning-based IDGA method was applied. Support Vector Machine (SVM), Naïve Bayes (NB), and K-Nearest Neighbour (KNN) are employed as classifiers on five microarray cancer datasets. The results showed that when combined with the IDGA, Fisher's score outperformed the Laplacian score on four datasets.

A hybrid framework that employs both extraction and wrapper gene selection methods was developed in [20]; as an extraction method, the authors used ICA, while as a wrapper method, ABC was utilized. A Gene Selection Programming (GSP) method was proposed in [21] to select relevant genes for cancer classification. GSP is a Gene Expression Programming (GEP) method with a newly defined population initialization algorithm and fitness function, improved mutation, and recombination operators. The authors adopted the SVM with a linear kernel as a classifier for the GSP. A neighborhood entropy-based feature selection algorithm was introduced in [22]. At first, the neighborhood entropy-based uncertainty measures were utilized to assess the uncertainty and to exclude the noise present in gene expression datasets. In the subsequent steps, the neighborhood credibility degree and coverage degree were applied. The authors employed a heuristic reduction algorithm to decrease the computational complexity and to improve the classification performance.

A hybrid method that employs Adaptive Elastic Net (AEN) with Conditional Mutual Information (CMI) was introduced in [23]. AEN-CMI obtained better performance compared to L1-SVM, Elastic Net, Adaptive Lasso, and classic Adaptive Elastic Net algorithms on two cancer microarray datasets. In [24], an unsupervised two-stage feature selection technique was presented. In the first stage, three filter-based methods techniques were applied. A genetic algorithm was utilized in the second stage. Finally, three ML classifiers, SVM, $\mathrm{k}-\mathrm{NN}$, and random forest (RF) were employed for classification.

Compared to the existing works, our method differs in the way how we incorporate various feature selection and classification techniques. Among the closely related PSO-based 
methods ([25-27]), HPSO-LS [25] embedded a local search strategy within the PSO instead of exercising correlation analysis to reduce the feature space before applying PSO. Besides, they used the Pearson correlation coefficient to identify correlation among features that can not capture the non-linear relationship. As a classifier, they used the k-NN classifier instead of SVM. In another PSO-based work [26], k-NN with PSO was utilized for feature selection and classification. However, their work focused more on selecting the best $k$ value for the k-NN classifier to achieve better accuracy. In [27], the correlation-based feature selection (CFS) algorithm and iBPSO were applied for identifying the best feature set, and then NB classifiers were employed for classification.

\subsection{Particle Swarm Optimization (PSO)}

Particle Swarm Optimization (PSO) [28] is a bio-inspired stochastic optimization algorithm that iteratively optimizes a set of candidate solutions (i.e., particles) in a swarm. Each particle moves in a multi-dimensional search space based on its velocity. Each particle's movement (i.e., velocity) is influenced by its local best-known position, $p$ Best and global best position, gBest (determined by all the particles in the swarm). PSO assumes that $g B e s t$ directs the swarm toward the best solutions. The following equations are used to update the position of particle $i$ in dimension $d$ -

$$
\begin{array}{r}
v_{i d}(t+1)=\omega_{i}(t) * v_{i d}(t)+c_{1} * r_{1} *\left(\text { pBest }_{i d}-x_{i d}(t)\right) \\
+c_{2} * r_{2} *\left(\text { gBest }_{d}-x_{i d}(t)\right) \\
x_{i d}(t+1)=x_{i d}(t)+v_{i d}(t) \\
\omega_{i}=\omega_{M a x}-l *\left(\left(\omega_{\text {Max }}-\omega_{\text {Min }}\right) / \text { total iteration }\right)
\end{array}
$$

In the above equations, $v_{i d}(t)$ and $x_{i d}(t)$ represent the velocity and position of particle $i$ in dimension $d$ at time $t$, respectively, while $v_{i d}(t+1)$ and $x_{i d}(t+1)$ are the updated velocity and position in $d^{\prime}$ th dimension of particle $i$ at time $(t+1) ; c 1$ and $c 2$ are arbitrary constants, and $r 1$ and $r 2$ are random variables $(0,1)$ of uniform distribution. $p$ Best $t_{i d}$ is the best solution of particle $i$ for $d^{\prime}$ th dimension at time $t$, while $g_{B} e s t_{d}$ is the overall global best solution in $d$ 'th dimension among all the particles at time t. The $p B e s t_{i d}$ of a particle is used for exploitation, while $g B e s t_{d}$ helps exploration of the solution space. This way particles try to reach closer to both their own best solution and the global best solution with a certain probability and weight factor. $\omega$ is a linearly decreasing inertia factor that gives less weight to the previous velocity over the period of iterations $(l)[29]$ and total iteration is the number of iterations used in PSO to update particles positions. An upper bound $x_{\max }$ and a lower bound $x_{m i n}$ are considered to make sure that particles remain in a finite search space. The position of a particle $i$ at time $(\mathrm{t}+1)$ is computed based on equation 2.2.

\subsection{Binary Particle Swarm Optimization (BPSO)}

We use a modified version of particle swarm optimization, called binary particle swarm optimization (BPSO) [30] for feature selection. BPSO sets the feature positions of a particle based on the discrete values of binary ' 0 ' and ' 1 ' instead of continuous values. A Sigmoid function is used to map the continuous valued velocity given by Eq. 2.1 to the range $[0,1]$, as shown in Eq. 2.4.

$$
\operatorname{sig}\left(v_{i d}\right)=\frac{1}{1+\exp \left(-v_{i d}\right)}
$$


The feature states (i.e., positions) of a particle are changed based on the following equations. For example, the state of the $d$ 'th feature in particle $i$ at time $t$ is determined by,

$$
x_{i d}(t)= \begin{cases}0 & \text { if } \rho_{i d} \geq \operatorname{sig}\left(v_{i d}\right) \\ 1, & \text { otherwise }\end{cases}
$$

where $\rho_{i d}$ is a random number with uniform distribution.

If $\operatorname{sig}\left(v_{i d}\right)$ is larger than the random value $\rho_{i d}$, then its state (position) is set to 1 (which refers the feature $d$ of the solution $i$ has been selected). On the other hand, if $\operatorname{sig}\left(v_{i d}\right)$ is smaller than or equal to the $\rho_{i d}$, its position is set as 0 , which means that the feature $d$ is not picked.

\section{Proposed Methodology}

The proposed methodology consists of several steps as shown in Figure 1.

\subsection{Feature Scaling}

Gene expression profiles often exhibit a skewed distribution of the gene expression values. The variation in gene expression can affect the performance of the classifier. To circumvent this, we perform min-max scaling to fit the value of each gene within a given range.

We scale each feature (i.e., gene) within a range between 0 and 1 . The following equation is used to normalize the $d^{\prime}$ 'th gene expression value of feature $i$ in set $X$,

$$
X_{i d} . s c a l e d=\frac{\left(X_{i d}-X_{i} \cdot \min \right)}{X_{i} \cdot \max -X_{i} \cdot \min }
$$

where, $X_{i}$.max and $X_{i}$.min are the maximum and minimum gene expression values of gene $i$, respectively.

\subsection{Feature Selection: Phase 1}

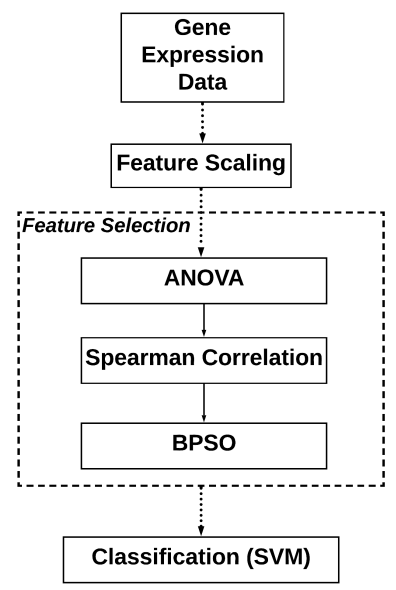

Figure 1. The Flowchart of the proposed methodology

Due to the presence of thousands of genes in microarray datasets, employing BPSO without reducing feature space is not a feasible approach. Therefore, in phase 1, we apply the ANOVA, a statistical method, to reduce the search space by selecting the top informative 
genes. ANOVA F-test compares the means of two or more groups to find whether they are significantly different from each other. ANOVA F-test determines the variance between the groups and variance within groups, calculates F-value, and uses it to identify informative genes. The selected class-correlated genes in this step are passed to phase 2 .

\subsection{Feature Selection: Phase 2}

The univariate filter-based feature selection methods rank features independently without considering the dependency among them; therefore, they can select highly informative but redundant genes. The redundant genes affect the performance of the classifier negatively, despite being class discriminative. In phase 2, we eliminate redundant genes identified in phase 1 using Spearman rank-order correlation.

The Spearman rank-order correlation coefficient (Spearman's $\rho$ ) is a non-parametric measure of the monotonicity of the relationship between two variables. It varies between -1 and +1 , where +1 or -1 occurs when one of the variables is a perfect monotone function of the other, while 0 implies no correlation. In contrast to Pearson correlation, which only assesses linear relationships between variables, Spearman's $\rho$ can capture both linear and non-linear relationships; therefore, more suitable for finding feature-feature correlation.

Let $k$ is the sample size and the $k$ raw scores of two variables $X$ and $Y$ are $X_{1}, \ldots, X_{k}$ and $Y_{1}, \ldots . . Y_{k}$, respectively. The ranks of $X$ and $Y$ are represented by $R_{x}\left(X_{1}, \ldots ., X_{k}\right)$ and $R_{y}\left(Y_{1}, \ldots, Y_{k}\right)$, respectively. The following formula is used to calculate the Spearman's $\rho$ :

$$
\rho=1-\frac{6 \sum d_{i}^{2}}{k\left(k^{2}-1\right)}
$$

where, $\rho=$ Spearman rank correlation, $d_{i}=R_{x}\left(X_{i}\right)-R_{y}\left(Y_{i}\right)$, the difference between the ranks of each observation, $k=$ number of observations

We calculate Spearman's $\rho$ between all pairs of features selected in phase 1. As the high correlation between two features refers to redundancy, we eliminate the lower-ranked (based on the class-correlation score computed in phase 1) feature. Variables numbers of genes are discarded in this step by varying the Spearman rank correlation coefficient threshold. Note that distinct threshold values are selected for various datasets, which are determined empirically.

\subsection{Feature Selection: Phase 3}

Phase 3 of the feature selection step leverages binary particle swarm optimization (BPSO) and SVM classifier. The BPSO optimization process involves several steps, as shown in Fig 2. We use the BPSO implementation of [31].

\subsubsection{Defining objective function}

The first step of BPSO is to define the fitness function to identify the optimal feature set. Since our goal is to identify a feature set that provides the highest accuracy, the optimization function is set accordingly. We do not consider minimizing the number of genes as an optimization goal since irrelevant genes are discarded in the previous steps.

\subsubsection{Classifier selection}

To calculate the accuracy of various feature sets generated by BPSO, a classifier needs to be employed. SVM classifier has been successfully utilized by many researchers for cancer classification [32,33]. We use $90 \%$ data to train the classifier and the remaining $10 \%$ for testing. The data split is performed randomly and in a stratified fashion. 


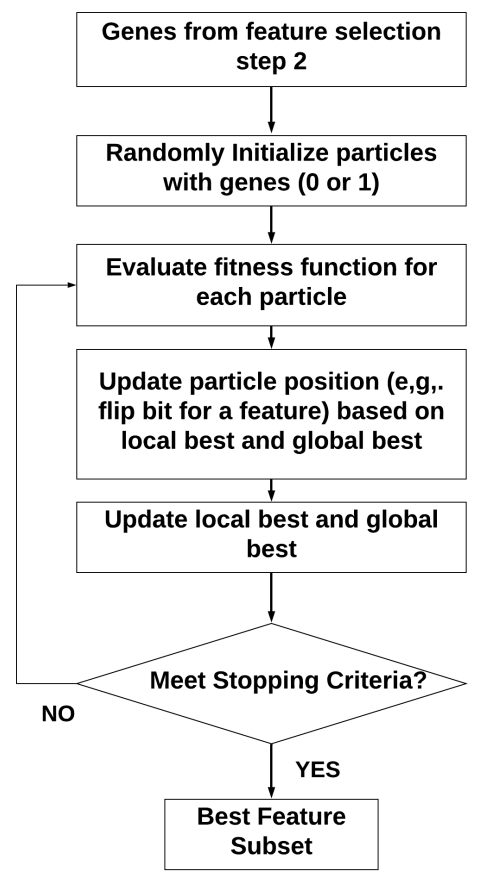

Figure 2. The steps of BPSO

\subsubsection{Particle initialization}

The initialization phase populates the swarm by creating a population of $\mathrm{n}$ particles (i.e., candidate solutions). Each particle represents an $d$-dimensional feature (i.e., gene) set, where $g_{i}=1$ means the gene $i$ is selected, while 0 value means it is not used. Initially, each particle position is set randomly, which means gene values are randomly set (0 or 1$)$.

\subsubsection{Finding the (sub)optimal solution}

In this step, BPSO searches for the best feature set by iteratively updating $x_{i d}$ based on equations 2.4 and 2.5. The fitness function is computed for each set, and gradually all the feature sets move towards the global best solution. This process terminates when no significant improvement for gBest is observed for 20 iterations with a maximum iteration limit set to 100 (derived empirically). Then we extract the feature set having the best solution and utilize it in the classification stage. The following parameter values are used for binary PSO algorithm, swarm size $=100, \omega_{\max }=0.9, \omega_{\min }=0.4, c 1=2, c 2=2$, which are chosen empirically.

\subsection{Classification}

Finally, in the classification stage, we apply the SVM classifier for identifying cancer types utilizing the genes selected in the feature selection stages. We use the default parameters settings of the SVM classifier and report the 10-fold cross-validation accuracy. We use the scikit-learn [34] implementation of SVM classifier. 


\section{Dataset and Baselines}

\subsection{Gene Expression Datasets}

We report the performance of ANOVA-SRC-BPSO on seven gene expression benchmark datasets, shown in Table 1. The datasets represent gene expression profiles of different types of cancers, such as Colon [35], Central Nerve System (CNS) [36], DLBCL [37], Leukemia [38], Lung [39], Prostate [40], and SRBCT [41]. Most of the datasets consist of binary classes; only the SRBCT dataset contains four classes.

The Colon dataset is a binary dataset consisting of gene expression profiles of 40 cancerous and 22 normal colon tissues. The CNS dataset comprises 61 examples from two classes. The DLBCL dataset consists of 77 gene expression samples from two classes, DLBCL and Follicular Lymphoma (FL) morphology. 72 samples (25 AML and 47 ALL) exist in the Leukemia dataset. Lung cancer consists of 181 samples, 31 from Malignant Pleural Mesothelioma (MPM) and 150 from Adenocarcinoma (ADCA). The Prostate and SRBCT datasets contain 102 and 63 samples, respectively. The four classes of the SRBCT dataset are neuroblastoma (NB), rhabdomyosarcoma (RMS), non-Hodgkin lymphoma (NHL), and the Ewing family of tumors (EWS).

Table 1. Gene Expression Datasets

\begin{tabular}{c|c|c|c|} 
Dataset & \#Gene & \#Sample & Class Distribution \\
& & & \\
Colon & 2000 & 62 & $2(22 / 40)$ \\
Central Nervous System(CNS) & 7129 & 61 & $2(21 / 40)$ \\
DLBCL & 7128 & 77 & $2(58 / 19)$ \\
Leukemia & 7129 & 72 & $2(47 / 25)$ \\
Lung & 12533 & 181 & $2(31 / 150)$ \\
Prostate & 10509 & 102 & $2(52 / 50)$ \\
SRBCT & 2308 & 63 & $4(23 / 20 / 12 / 8)$
\end{tabular}

\subsection{Baseline Methods}

The efficacy of ANOVA-SRC-BPSO is shown by comparing it with fourteen state-ofthe-art methods. The summary of each method is provided in Table 2. Most of them use bio-inspired optimization algorithms [18-20, 24-27, 42, 43].

Table 2. State-of-the-arts methods

\begin{tabular}{c|c} 
Baseline & Feature Selection and Classification methods \\
Li[33] & Fisher-Markov, Multi-objective Binary Biogeography Based Optimization (MOBBBO), SVM \\
Shreem[44] & Harmony Search Algorithm (HSA), Markov Blanket (MB), NB \\
Nguyen[45] & Hidden Markov Models (HMMs), Modified Analytic Hierarchy Process (AHP) \\
Aziz[20] & Independent Component Analysis (ICA), Artificial Bee Colony (ABC), NB \\
Dashtban[19] & Laplacian and Fisher score, Intelligent Dynamic Genetic Algorithm, SVM, KNN, NBY \\
Salem[18] & Information Gain (IG), Standard Genetic Algorithm (SGA), Genetic Programming (GP) \\
Sun[22] & Neighborhood Entropy-based Uncertainty Measures \\
Wang[23] & Adaptive Elastic Net, Conditional Mutual Information (AEN-CMI) \\
Al-Obeidat[24] & PCA, Correlation and Spectral-based Feature Selection, Genetic Algorithm (GA), SVM, K-NN, RF. \\
Alshamlan[42] & Artificial Bee Colony (ABC), SVM \\
Moradi [25] & PSO, Local Search \\
Alomari[43] & Minimum Redundancy Maximum Relevancy (MRMR), Bat-inspired Algorithm (BA), SVM \\
Kar[26] & PSO, adaptive K-nearest neighborhood \\
Jain[27] & Correlation-based Feature Selection (CFS), improved-Binary PSO (iBPSO), Naive-Bayes (NB) \\
&
\end{tabular}




\section{Experimental Setup and Results}

\subsection{Performance Metrics}

We compare the performance of ANOVA-SRC-BPSO with state-of-the-art methods in terms of accuracy and the number of genes utilized for classification. The accuracy is defined as follows-

$$
\text { Accuracy }=\frac{\text { Number of samples correctly classified in a dataset }}{\text { Total number of samples present in the dataset }}
$$

Besides, we investigate the number of genes different methodologies use for classification.

\subsection{Comparative Results}

Table 3 shows the comparison results of ANOVA-SRC-BPSO with a number of state-ofthe-art methods. Both accuracy and number of genes used by various methods are compared (except [24, 45], which did not report the number of genes utilized). In the Colon dataset, among various methods, [20] shows the highest accuracy of 0.921 using 4 genes, while ANOVA-SRC-BPSO delivers an accuracy of 0.919 using 18 genes. In the CNS dataset, the ANOVA-SRC-BPSO provides an accuracy of 0.967 using 44 genes, while other methods provide lower accuracy using varied numbers of genes. In the DLBCL dataset, the ANOVASRC-BPSO reaches $100 \%$ accuracy with 6 genes, two other methods, [33] and [19], also achieve $100 \%$ accuracy using 5.7 and 9 genes, respectively.

In the Leukemia dataset, ANOVA-SRC-BPSO reaches an accuracy of 0.988 using 6 genes; among the other methods, [44] provides an accuracy of 0.993 using 5 genes. [19] shows an accuracy of 1.0 using 15 genes. In the Lung dataset, ANOVA-SRC-BPSO yields an accuracy of 0.994. Among the other works, [24] shows similar accuracy, while other methods yield lower accuracy.

In the Prostate cancer dataset, ANOVA-SRC-BPSO delivers an accuracy of $0.971 \mathrm{em}-$ ploying 9 genes. Although, [19], [18], and [33] provide higher accuracy, they use 14, 11.9 and 26 genes, respectively. The only framework that utilizes a small number of genes is [20], which yields lower accuracy of 0.892 compared to the proposed approach. In the SRBCT dataset, ANOVA-SRC-BPSO obtains an accuracy of 1.0 using only 6 genes. Among other methods, [19] and [33] obtain perfect accuracy of 1.0; however, they use 18 and 6.4 genes, respectively.

Table 3. The comparison of accuracy and genes utilized by various methods in seven gene expression datasets

\begin{tabular}{c|c|c|c|c|c|c|c|} 
Method & Colon & CNS & DLBCL & Leukemia & Lung & Prostate & SRBCT \\
Wang [3] & $0.89(25.20)$ & - & - & $0.91(26.85)$ & - & - & - \\
Dashban [4] & - & - & $\mathbf{1 . 0 0 ( 9 )}$ & $\mathbf{1 . 0 ( 1 5 )}$ & - & $0.96(14)$ & $\mathbf{1 . 0}(\mathbf{1 8})$ \\
Ngyuen [5] & 0.89 & - & 0.99 & 0.98 & - & 0.92 & - \\
Li [6] & - & - & $\mathbf{1 . 0 0 ( 5 . 7 )}$ & - & - & $0.98(11.9)$ & $\mathbf{1 . 0 ( 6 . 4 )}$ \\
Aziz [7] & $0.92(4)$ & - & - & $0.94(6)$ & $0.84(4)$ & $0.89(4)$ & - \\
Al-obeidat [8] & 0.85 & 0.85 & 0.98 & 0.90 & 0.99 & 0.99 & - \\
Sheerm [9] & $0.90(4.16)$ & $0.84(7.43)$ & - & $0.99(5)$ & 0.96 & - & $0.99(8.9)$ \\
Salem [10] & $0.85(60)$ & $0.87(38)$ & $0.95(110)$ & $0.97(3)$ & - & $\mathbf{1 . 0 ( 2 6 )}$ & - \\
Sun [11] & $0.84(3)$ & - & $0.93(11)$ & $0.93(9)$ & $0.99(8)$ & - & $0.94(9)$ \\
Alshamlan [12] & $0.92(20)$ & - & - & - & $0.93(8)$ & $0.92(10)$ & - \\
Moradi [13] & - & - & - & $0.89(100)$ & - & - & - \\
Alomari [14] & $0.93(8.13)$ & $0.94(19.2)$ & - & $\mathbf{1 . 0 ( 4 . 3 )}$ & - & - & $\mathbf{1 . 0}(\mathbf{1 2 . 8 3})$ \\
Kar [15] & - & - & - & $0.97(2.7)$ & - & - & $0.96(8.5)$ \\
Jain [16] & $\mathbf{0 . 9 5 ( 4 . 2 )}$ & $0.96(10.5)$ & - & $\mathbf{1 . 0 ( 4 . 3 )}$ & $\mathbf{1 . 0 ( 6 )}$ & - & $\mathbf{1 . 0}(\mathbf{3 4 . 1})$ \\
Proposed & $0.92(18)$ & $\mathbf{0 . 9 7 ( 4 4 )}$ & $\mathbf{1 . 0 ( 6 )}$ & $\mathbf{1 . 0 ( 2 3 )}$ & $\mathbf{1 . 0 ( 3 )}$ & $0.97(9)$ & $\mathbf{1 . 0 ( 6 )}$
\end{tabular}




\section{Discussion}

The comparisons with the state-of-the-art methods demonstrate that ANOVA-SRCBPSO provides comparable accuracy. In several datasets, such as DLBCL, Lung, and SRBCT datasets, ANOVA-SRC-BPSO achieves the highest accuracy using only a small number of genes (less than 6). Among the seven datasets, ANOVA-SRC-BPSO yields perfect accuracy in 4 datasets. Yet, in several datasets, we observe that few of the existing methods perform slightly better; However, in most cases, the improvement is not significant, or a higher number of genes are utilized. For example, in the Prostate cancer dataset, [18] using 26 genes achieves an accuracy of 1.00, while ANOVA-SRC-BPSO uses 9 genes to achieve 0.971 . The comparison results reveal that no single method (including the proposed ANOVA-SRC-BPSO) always performs better than the others. The accuracy of various methods depends on the dataset, the number of genes utilized and the parameters used.

\section{Conclusion}

In this study, we propose a hybrid cancer classification framework that utilizes ANOVA for selecting the initial set of class-discriminative genes, which are further examined for feature redundancy using Spearman's correlation. Finally, we employ BPSO and SVM to identify an optimized feature set to identify cancer subtypes. We observe among the seven gene expression datasets, ANOVA-SRC-BPSO attains 100\% classification accuracy on 4 datasets using a varied number of genes. The comparisons with the fourteen state-of-theart methods on seven microarray datasets demonstrate that ANOVA-SRC-BPSO exhibits comparable performance in terms of classification accuracy and the number of genes utilized. The results demonstrate that ANOVA-SRC-BPSO is a highly effective approach for feature selection in the microarray dataset. The comparative analysis also reveals that although several methods achieve perfect accuracy in multiple gene expression datasets, there is still a need for developing methods that exhibit consistent performances across datasets.

\section{References}

[1] R. Ge, M. Zhou, Y. Luo, Q. Meng, G. Mai, D. Ma, G. Wang, and F. Zhou. "McTwo: a two-step feature selection algorithm based on maximal information coefficient". In: BMC bioinformatics 17.1 (2016), pp. 1-14.

[2] A. Jain and D. Zongker. "Feature selection: Evaluation, application, and small sample performance". In: IEEE transactions on pattern analysis and machine intelligence 19.2 (1997), pp. 153-158.

[3] Y. Leung and Y. Hung. "A multiple-filter-multiple-wrapper approach to gene selection and microarray data classification". In: IEEE/ACM Transactions on Computational Biology and Bioinformatics 7.1 (2008), pp. 108-117.

[4] I. Guyon and A. Elisseeff. "An introduction to variable and feature selection". In: Journal of machine learning research 3.Mar (2003), pp. 1157-1182.

[5] H. Liu and R. Setiono. "Chi2: Feature selection and discretization of numeric attributes". In: Proceedings of rth IEEE International Conference on Tools with Artificial Intelligence. IEEE. 1995, pp. 388-391.

[6] C.-M. Lai, W.-C. Yeh, and C.-Y. Chang. "Gene selection using information gain and improved simplified swarm optimization". In: Neurocomputing 218 (2016), pp. 331-338.

[7] L. Gao, M. Ye, X. Lu, and D. Huang. "Hybrid method based on information gain and support vector machine for gene selection in cancer classification". In: Genomics, proteomics 86 bioinformatics 15.6 (2017), pp. 389-395.

[8] H. Xiaofei, C. Deng, and N. Partha. "Laplacian score for feature selection". In: Advances in neural information processing systems (2005), pp. 507-514. 
[9] S. Dudoit, J. Fridlyand, and T. P. Speed. "Comparison of discrimination methods for the classification of tumors using gene expression data". In: Journal of the American statistical association 97.457 (2002), pp. 77-87.

[10] H. Peng, F. Long, and C. Ding. "Feature selection based on mutual information criteria of max-dependency, max-relevance, and min-redundancy". In: IEEE Transactions on pattern analysis and machine intelligence 27.8 (2005), pp. 1226-1238.

[11] C. Lai, M. J. Reinders, and L. Wessels. "Random subspace method for multivariate feature selection". In: Pattern recognition letters 27.10 (2006), pp. 1067-1076.

[12] A. J. Ferreira and M. A. Figueiredo. "An unsupervised approach to feature discretization and selection". In: Pattern Recognition 45.9 (2012), pp. 3048-3060.

[13] S. S. Shreem, S. Abdullah, M. Z. A. Nazri, and M. Alzaqebah. "Hybridizing ReliefF, MRMR filters and GA wrapper approaches for gene selection". In: J. Theor. Appl. Inf. Technol 46.2 (2012), pp. 1034-1039.

[14] L.-Y. Chuang, C.-H. Yang, K.-C. Wu, and C.-H. Yang. "A hybrid feature selection method for DNA microarray data". In: Computers in biology and medicine 41.4 (2011), pp. 228-237.

[15] H. Alshamlan, G. Badr, and Y. Alohali. "mRMR-ABC: a hybrid gene selection algorithm for cancer classification using microarray gene expression profiling". In: Biomed research international 2015 (2015).

[16] H. Lu, J. Chen, K. Yan, Q. Jin, Y. Xue, and Z. Gao. "A hybrid feature selection algorithm for gene expression data classification". In: Neurocomputing 256 (2017), pp. 56-62.

[17] F. V. Sharbaf, S. Mosafer, and M. H. Moattar. "A hybrid gene selection approach for microarray data classification using cellular learning automata and ant colony optimization". In: Genomics 107.6 (2016), pp. 231-238.

[18] H. Salem, G. Attiya, and N. El-Fishawy. "Classification of human cancer diseases by gene expression profiles". In: Applied Soft Computing 50 (2017), pp. 124-134.

[19] M Dashtban and M. Balafar. "Gene selection for microarray cancer classification using a new evolutionary method employing artificial intelligence concepts". In: Genomics 109.2 (2017), pp. 91-107.

[20] R. Aziz, C. Verma, and N. Srivastava. "A novel approach for dimension reduction of microarray". In: Computational biology and chemistry 71 (2017), pp. 161-169.

[21] R. Alanni, J. Hou, H. Azzawi, and Y. Xiang. "A novel gene selection algorithm for cancer classification using microarray datasets". In: BMC medical genomics 12.1 (2019), p. 10.

[22] L. Sun, X. Zhang, Y. Qian, J. Xu, and S. Zhang. "Feature selection using neighborhood entropy-based uncertainty measures for gene expression data classification". In: Information Sciences 502 (2019), pp. 18-41.

[23] Y. Wang, X.-G. Yang, and Y. Lu. "Informative gene selection for microarray classification via adaptive elastic net with conditional mutual information". In: Applied Mathematical Modelling 71 (2019), pp. 286-297.

[24] F. Al-Obeidat, A. Tubaishat, B. Shah, Z. Halim, et al. "Gene encoder: a feature selection technique through unsupervised deep learning-based clustering for large gene expression data". In: Neural Computing and Applications (2020), pp. 1-23.

[25] P. Moradi and M. Gholampour. "A hybrid particle swarm optimization for feature subset selection by integrating a novel local search strategy". In: Applied Soft Computing 43 (2016), pp. $117-130$.

[26] S. Kar, K. D. Sharma, and M. Maitra. "Gene selection from microarray gene expression data for classification of cancer subgroups employing PSO and adaptive K-nearest neighborhood technique". In: Expert Systems with Applications 42.1 (2015), pp. 612-627.

[27] I. Jain, V. K. Jain, and R. Jain. "Correlation feature selection based improved-binary particle swarm optimization for gene selection and cancer classification". In: Applied Soft Computing 62 (2018), pp. 203-215.

[28] J. Kennedy and R. Eberhart. "Particle swarm optimization". In: Proceedings of ICNN'95international conference on neural networks. Vol. 4. IEEE. 1995, pp. 1942-1948.

[29] Y. Shi and R. C. Eberhart. "Fuzzy adaptive particle swarm optimization". In: Proceedings of the 2001 congress on evolutionary computation (IEEE Cat. No. 01TH8546). Vol. 1. IEEE. 2001, pp. 101-106. 
[30] J. Kennedy and R. C. Eberhart. "A discrete binary version of the particle swarm algorithm". In: 1997 IEEE International conference on systems, man, and cybernetics. Computational cybernetics and simulation. Vol. 5. IEEE. 1997, pp. 4104-4108.

[31] R. A. Khurma, I. Aljarah, A. Sharieh, and S. Mirjalili. "Evolopy-fs: An open-source natureinspired optimization framework in python for feature selection". In: Evolutionary Machine Learning Techniques. Springer, 2020, pp. 131-173.

[32] H. M. Alshamlan, G. H. Badr, and Y. A. Alohali. "Genetic Bee Colony (GBC) algorithm: A new gene selection method for microarray cancer classification". In: Computational biology and chemistry 56 (2015), pp. 49-60.

[33] X. Li and M. Yin. "Multiobjective binary biogeography based optimization for feature selection using gene expression data". In: IEEE Transactions on NanoBioscience 12.4 (2013), pp. 343-353.

[34] F. Pedregosa, G. Varoquaux, A. Gramfort, V. Michel, B. Thirion, O. Grisel, M. Blondel, P. Prettenhofer, R. Weiss, V. Dubourg, J. Vanderplas, A. Passos, D. Cournapeau, M. Brucher, M. Perrot, and E. Duchesnay. "Scikit-learn: Machine Learning in Python". In: Journal of Machine Learning Research 12 (2011), pp. 2825-2830.

[35] U. Alon, N. Barkai, D. A. Notterman, K. Gish, S. Ybarra, D. Mack, and A. J. Levine. "Broad patterns of gene expression revealed by clustering analysis of tumor and normal colon tissues probed by oligonucleotide arrays". In: Proceedings of the National Academy of Sciences 96.12 (1999), pp. 6745-6750.

[36] S. L. Pomeroy, P. Tamayo, M. Gaasenbeek, L. M. Sturla, M. Angelo, M. E. McLaughlin, J. Y. Kim, L. C. Goumnerova, P. M. Black, C. Lau, et al. "Prediction of central nervous system embryonal tumour outcome based on gene expression". In: Nature 415.6870 (2002), pp. 436-442.

[37] M. A. Shipp, K. N. Ross, P. Tamayo, A. P. Weng, J. L. Kutok, R. C. Aguiar, M. Gaasenbeek, M. Angelo, M. Reich, G. S. Pinkus, et al. "Diffuse large B-cell lymphoma outcome prediction by gene-expression profiling and supervised machine learning". In: Nature medicine 8.1 (2002), pp. $68-74$.

[38] T. R. Golub, D. K. Slonim, P. Tamayo, C. Huard, M. Gaasenbeek, J. P. Mesirov, H. Coller, M. L. Loh, J. R. Downing, M. A. Caligiuri, et al. "Molecular classification of cancer: class discovery and class prediction by gene expression monitoring". In: science 286.5439 (1999), pp. $531-537$.

[39] G. J. Gordon, R. V. Jensen, L.-L. Hsiao, S. R. Gullans, J. E. Blumenstock, S. Ramaswamy, W. G. Richards, D. J. Sugarbaker, and R. Bueno. "Translation of microarray data into clinically relevant cancer diagnostic tests using gene expression ratios in lung cancer and mesothelioma". In: Cancer research 62.17 (2002), pp. 4963-4967.

[40] D. Singh, P. G. Febbo, K. Ross, D. G. Jackson, J. Manola, C. Ladd, P. Tamayo, A. A. Renshaw, A. V. D'Amico, J. P. Richie, et al. "Gene expression correlates of clinical prostate cancer behavior". In: Cancer cell 1.2 (2002), pp. 203-209.

[41] J. Khan, J. S. Wei, M. Ringner, L. H. Saal, M. Ladanyi, F. Westermann, F. Berthold, M. Schwab, C. R. Antonescu, C. Peterson, et al. "Classification and diagnostic prediction of cancers using gene expression profiling and artificial neural networks". In: Nature medicine 7.6 (2001), pp. 673-679.

[42] H. M. Alshamlan, G. H. Badr, and Y. A. Alohali. "Abc-svm: artificial bee colony and svm method for microarray gene selection and multi class cancer classification". In: Int. J. Mach. Learn. Comput 6.3 (2016), p. 184.

[43] O. A. Alomari, A. T. Khader, M. A. Al-Betar, and L. M. Abualigah. "Gene selection for cancer classification by combining minimum redundancy maximum relevancy and bat-inspired algorithm". In: International Journal of Data Mining and Bioinformatics 19.1 (2017), pp. 3251.

[44] S. S. Shreem, S. Abdullah, and M. Z. A. Nazri. "Hybridising harmony search with a Markov blanket for gene selection problems". In: Information Sciences 258 (2014), pp. 108-121.

[45] T. Nguyen, A. Khosravi, D. Creighton, and S. Nahavandi. "Hidden Markov models for cancer classification using gene expression profiles". In: Information Sciences 316 (2015), pp. 293307. 